\title{
Exercise-induced angiogenesis correlates with the up-regulated expression of neuronal nitric oxide synthase (nNOS) in human skeletal muscle
}

\author{
Felicitas A. M. Huber-Abel • Mélanie Gerber • \\ Hans Hoppeler · Oliver Baum
}

Received: 1 November 2010 / Accepted: 4 April 2011 / Published online: 20 April 2011

(C) Springer-Verlag 2011

\begin{abstract}
The contribution of neuronal nitric oxide synthase (nNOS) to angiogenesis in human skeletal muscle after endurance exercise is controversially discussed. We therefore ascertained whether the expression of nNOS is associated with the capillary density in biopsies of the vastus lateralis (VL) muscle that had been derived from 10 sedentary male subjects before and after moderate training (four 30-min weekly jogging sessions for 6 months, with a heart-rate corresponding to $75 \% \mathrm{VO}_{2} \max$ ). In these biopsies, nNOS was predominantly expressed as alpha-isoform with exon-mu and to a lesser extent without exon-mu, as determined by RT-PCR. The mRNA levels of nNOS were quantified by real-time PCR and related to the capillary-tofibre ratio and the numerical density of capillaries specified by light microscopy. If the VL biopsies of all subjects were co-analysed, mRNA levels of nNOS were non-significantly elevated after training $(+34 \% ; P>0.05)$. However, only five of the ten subjects exhibited significant $(P \leq 0.05)$ elevations in the capillary-to-fibre ratio $(+25 \%)$ and the numerical density of capillaries $(+21 \%)$ and were thus undergoing angiogenesis. If the VL biopsies of these five subjects alone were evaluated, the mRNA levels of nNOS were significantly up-regulated $(+128 \% ; P \leq 0.05)$ and correlated positively $(r=0.8 ; P \leq 0.01)$ to angiogenesis. Accordingly, nNOS protein expression in VL biopsies quantified by immunoblotting was significantly increased $(+82 \% ; P \leq 0.05)$ only in those subjects that underwent angiogenesis. In conclusion, the expression of nNOS at
\end{abstract}

Communicated by Martin Flueck.

F. A. M. Huber-Abel · M. Gerber · H. Hoppeler · O. Baum ( $\square)$ Institute of Anatomy, University of Bern,

Baltzerstrasse 2, 3009 Bern, Switzerland

e-mail: oliver.baum@ana.unibe.ch
mRNA and protein levels was statistically linked to capillarity after exercise suggesting that nNOS is involved in the angiogenic response to training in human skeletal muscle.

Keywords Angiogenesis - Human exercise physiology · Neuronal nitric oxide synthase $\cdot$ Skeletal muscle

\section{Introduction}

Skeletal muscles adapt to endurance exercise with characteristic and coordinated changes in the expression levels of many genes, which subsequently lead to multifaceted alterations in muscular ultrastructure and function. There are several established hallmarks of endurance exercise (reviewed in Coffey and Hawley 2007; Hoppeler et al. 2007). The expression of mitochondrial genes and oxidative enzymes is up-regulated, whereas glycolytic enzymes are downregulated in the skeletal muscle fibres. This shift in metabolism is accompanied by a distinct increase in density of capillaries surrounding the muscle fibres due to angiogenesis (reviewed in Egginton 2009; Hudlicka and Brown 2009).

The molecular mechanisms underlying the exerciseinduced changes in skeletal muscle phenotype, particularly of the microvascular system, are only incompletely understood. Conceptually, information relating to the higher rates of muscle contractility is translated into an up-stream signalling event, which is subsequently transduced into the extracellular environment surrounding the muscle fibres.

Neuronal NO synthase (nNOS) is expressed in high concentrations at the sarcolemma of the muscle fibres, where it generates nitric oxide (NO), a diffusible gaseous radical with impact on many processes in skeletal muscle (reviewed in Stamler and Meissner 2001). As an autocrine 
signalling molecule, nNOS-derived NO promotes the relaxation of skeletal muscle fibres to counteract their contraction (Kobzik et al. 1994) and participates in the regulation of the redox state in skeletal muscle fibres (Da Silva-Azevedo et al. 2009; Jackson et al. 2007). As a paracrine signalling molecule, nNOS-derived NO controls the cGMP-dependent relaxation of vascular smooth muscle cells in skeletal muscle, thereby contributing to vasodilation (Lau et al. 2000; Melikian et al. 2009).

Several studies demonstrated that the availability of NO is a critical factor for the implementation of angiogenesis in skeletal muscle of rodents. Down-regulation of NOS activity by chemical inhibition reduced the growth of the capillary system in skeletal muscles of rats after chronic electric stimulation (Hudlicka et al. 2000). Furthermore, sprouting angiogenesis was abolished in nNOS-knockout-mice in response to muscle overload induced by extirpation of a functional agonist (Williams et al. 2006). In accordance with this role of nNOS as modulator of angiogenesis, nNOS protein levels and activity revealed to be increased in skeletal muscles of rodents after chronic exercise (Vassilakopoulos et al. 2003). In contrast, the regulation and functional impact of nNOS to angiogenesis in response to endurance exercise in skeletal muscle of humans has been controversially discussed (Frandsen et al. 2000; McConell et al. 2007).

Our group has previously conducted a study with unexercised male subjects undergoing a moderate training enrolled in a 6-month jogging program (Suter et al. 1995). Analysing biopsies of the vastus lateralis (VL) muscle by means of morphometry, this training regime evoked a heterogenous structural response. While the mitochondrial density was higher in all subjects after exercise, only some study participants showed an alteration in the muscular intracellular lipid content and capillarity (Suter et al. 1995). To ascertain whether the expression of neuronal nitric oxide synthase (nNOS) is associated with exercise-induced angiogenesis in human skeletal muscle, we now quantified the nNOS expression at the mRNA and protein levels in these VL muscle biopsies. Statistical evaluation of the sets of data revealed the mRNA and protein levels of nNOS to be up-regulated in response to endurance exercise and to be correlated with the degree of angiogenesis. These findings suggest that nNOS is involved in the angiogenic response to training in skeletal muscle of humans.

\section{Materials and methods}

\section{Subjects and training protocol}

Fine-needle biopsies of the vastus lateralis (VL) muscle were derived from 10 male subjects in the context of an earlier study (Suter et al. 1995). Previous to the study, the participants had not been engaged in endurance activities. The moderate, home-based training regime consisted of four 30-min jogging sessions per week for 6 months, with a heart-rate corresponding to a maximal oxygen uptake $\left(\mathrm{VO}_{2} \max \right)$ of $75 \%$. The heart rate of the subjects participating in this study was continuously recorded during the training with a portable heart rate monitor as previously reported (Suter et al. 1995). $\mathrm{VO}_{2} \max$ was determined in a continuous incremental exercise test on a bicycle ergometer (start at $75 \mathrm{~W}$, followed by increments of $30 \mathrm{~W}$ every $2 \mathrm{~min}$ ) to voluntary exhaustion. The study conformed with the guidelines delineated by the Bernese Ethical Commission, and was conducted with its approval.

The male subjects had a mean age of $36.3( \pm 6.3)$ years, a mean height of $181.0( \pm 7.1) \mathrm{cm}$ and a mean weight of $85.6( \pm 16.0) \mathrm{kg}$ and a mean body-mass index of 26.2 $( \pm 4.0) \mathrm{kg} / \mathrm{cm}^{2}$. The mean $\mathrm{VO}_{2} \max$ increased from a pretraining value of $39.6( \pm 5.5) \mathrm{ml} / \mathrm{kg}$ min to a post-training one of $41.3( \pm 6.3) \mathrm{ml} / \mathrm{kg} \mathrm{min}$, but the difference was not significant $(P>0.05)$. VL biopsies, $50-100 \mathrm{mg}$ in weight, were obtained using a Bergström fine needle $48 \mathrm{~h}$ after the last training session, as previously described (Suter et al. 1995).

Morphometric analysis

$1-\mathrm{mm}^{3}$ pieces of the VL biopsies were chemically fixed for several days at $4{ }^{\circ} \mathrm{C}$ in an aqueous solution containing $2.5 \%$ $(\mathrm{v} / \mathrm{v})$ glutaraldehyde and 2\% (v/v) paraformaldehyde, which was buffered with $0.1 \mathrm{M}$ sodium cacodylate $(\mathrm{pH}$ 7.4) as previously described (Hoppeler et al. 1985; Suter et al. 1995). 1- $\mu \mathrm{m}$ thick sections for light microscopy were prepared and stained with toluidine blue to determine the capillary-to-fibre $(\mathrm{C} / \mathrm{F})$ ratio and the numerical density of capillaries on light micrographs (Baum et al. 2004). This approach represents the gold standard to quantitatively assess the capillarity due to the direct visualization of the microvasculature.

Transmission electron microscopy and morphometry to quantify the mitochondrial volume density and the mean fibre cross-sectional area was performed as previously described (Hoppeler et al. 1985).

\section{RNA extraction and reverse transcription}

For each biopsy, RNA was extracted from $10025-\mu \mathrm{m}$ thick cryo-sections using an RNeasy Mini kit (Qiagen, Hilden, Germany). The concentration of RNA was spectrophotometrically determined (absorbance wavelength: $260 \mathrm{~nm}$ ) using a NanoDrop (Thermo Scientific, Wilmington, DE, USA).

First-strand DNA was synthesized from the RNA using a Superscript VILO cDNA synthesis kit (Invitrogen, Basel, 
Switzerland) in accordance with the manufacturer's instructions.

\section{RT-PCR}

For the determination of the mRNA expression profiles of nNOS in human VL by reverse transcription (RT)-PCR, the GoTaq Hot Start Polymerase (Promega, Dübendorf, Switzerland) was used. This analysis was performed on six randomly selected biopsies taken prior to the exercise. nNOS exon-specific primer pairs were designed using Primer3 software and purchased from Microsynth (Balgach, Switzerland): exon 1a: 5'-CAG ATG GCA GCA GAC AGG TA-3'; exon 1c: 5'-CTG ACT GCC CTT GTC TCT CC-3'; exon 1f: 5'-CCC ACA CCC ATA AAC CAG TC-3'; exon $1 \mathrm{~g}$ : 5'-CGG GAG GAA GAG GAG GAG TA-3', exon 5 reverse: 5'-GGA GCC CATG CAG ATG TAC T-3'; exon 4-11: 5'-ACG CTT CCT CAA GGT CAA GA-3' and 5'-ACC GCG ATA TTG ATC TCC AC-3'; exon 10-15: 5'-CCC TTC AGT GGC TGG TAC AT-3' and 5'-TCA TGT TCC AGG TGC ACA AT-3'; exon 14-18: 5'-TAT GGC CAA GAG GGT GAA AG-3' and 5'-CGA GGC CAA AAA CTG AGA AC-3'; exon 17-22: 5'-ACA AGG TCC GAT TCA ACA GC-3' and 5'-CAG ACG CTG CTT CTC CTT CT-3'; exon 21-26: 5'-TGG TGA AAG TGG AAC TGC TG-3' and 5'-TGA AGA CCC CCT TGT TCT TG-3'; exon 25-28: 5'-CGG CAA TTT GAT ATC CAA CAC-3' and 5'-CTC AGA TCT AAG GCG GTT GG-3'. The total reaction volume of $25 \mu$ consisted of $5 \times$ Green GoTaq Buffer, dNTP mix (10 mM), $\mathrm{MgCl}_{2}$ solution (1 mM), primers $(100 \mu \mathrm{M})$, GoTaq Hot Start Polymerase (1.25 U), cDNA template (50 ng) and double-distilled water. The following PCR cycling protocol was applied: initial denaturation step at $94^{\circ} \mathrm{C}$ for $2 \mathrm{~min}$, then 35 cycles with denaturation at $94^{\circ} \mathrm{C}$ for $30 \mathrm{~s}$, annealing at $58^{\circ} \mathrm{C}$ for $60 \mathrm{~s}$, elongation at $72^{\circ} \mathrm{C}$ for $90 \mathrm{~s}$ and a final elongation step at $72^{\circ} \mathrm{C}$ for $10 \mathrm{~min}$. PCR fragments were visualized by $1 \%$ agarose gel electrophoresis supplemented with $0.5 \mu \mathrm{g} / \mathrm{ml}$ ethidium-bromide.

\section{Real-time PCR}

Real-time PCR was performed using the ABI Prism 7900 HT sequence detection system (Applied Biosystems, Rotkreuz, Switzerland) and SYBR Green PCR Master Mix for quantitative PCR (Applied Biosystems). Primers were designed using the software Primer Express (Applied Biosystems) and qPrimerDepot: nNOS primer pair: 5'-CAG CCC AAT GTC ATT TCT GTT-3' and 5'-GAT CAC GGG CGG CTT ACT-3'. For normalization, 18S rRNA levels were measured using the following primer pair: 5'-GCT TAA TTT GAC TCA ACA CGG GA-3' and 5'-AGC TAT CAA TCT GTC AAT CCT GTC-3'. The total reaction volume of $15 \mu \mathrm{l}$ consisted of $2 \times$ SYBR Green PCR Master Mix, primers (500 nM), cDNA (50 ng) and double-distilled water. Following a 15-min denaturation step at $95^{\circ} \mathrm{C}$, specific cDNAs were amplified through 40 cycles at $94^{\circ} \mathrm{C}$ for $15 \mathrm{~s}, 55^{\circ} \mathrm{C}$ for $30 \mathrm{~s}$ and $70^{\circ} \mathrm{C}$ for $30 \mathrm{~s}$. For recording of the dissociation curves the following protocol was used: $95^{\circ} \mathrm{C}$ for $15 \mathrm{~s}, 60^{\circ} \mathrm{C}$ for $15 \mathrm{~s}$ and $95^{\circ} \mathrm{C}$ for $15 \mathrm{~s}$. For analysis of the mRNA expression, cycling reports and melting curves were evaluated after setting of a baseline cycle threshold. Data were evaluated by the relative quantification method $\left(2^{-\Delta \mathrm{CT}}\right)$.

Immunoblotting

Immunoblotting was performed using $50 \mu \mathrm{g}$ of protein, as previously described (Da Silva-Azevedo et al. 2009). A polyclonal antibody (N-7280, Sigma), which specifically identifies amino acids 1409-1429 in the C-terminal region of nNOS, was incubated in a 1:10,000 dilution in washing buffer $(0.1 \%(\mathrm{w} / \mathrm{v})$ Tween 20 in PBS, pH 7.4) overnight at $4^{\circ} \mathrm{C}$.

\section{Statistics}

All numerical data are expressed as mean values ( \pm SEM). Parameters pertaining to the anthropometric and molecular analyses were compared using a paired Students $t$-test. Significance values relating to the real-time PCR data were calculated by a two-way ANOVA (repeated measures). The significance level was set at $P \leq 0.05$.

\section{Results}

mRNA expression profiles of nNOS isoforms in human VL biopsies

The mRNA expression profiles of nNOS isoforms were analysed in six randomly selected VL biopsies conducting RT-PCR. The 5'-UTR of the mRNA for nNOS was determined by combining an exon 5 reverse primer with forward primers derived from the four major mRNA variants of $5^{\prime}$-UTR (1a, 1c, 1f and 1g) that are generated by alternative splicing of the nNOS gene. When exon 1c was used as the upstream primer in the RT-PCR analysis, a single cDNA band (1,500 base-pairs) was detected. When any of the other three exon-variants (1a, 1f or $1 \mathrm{~g}$ ) were used as the forward primer, no cDNA was amplified (Fig. 1a). Nucleotide sequencing confirmed the 1c-2-3-4-5 exon-composition of the PCR-amplificate (data not shown).

Six additional pairs of exon-specific primers were designed, which covered the complete cDNA-coding sequence of nNOS downstream from exon 4. Using five of 
A

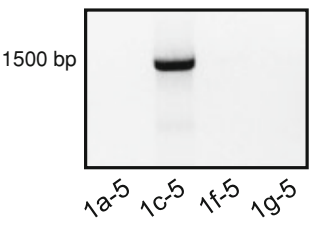

B

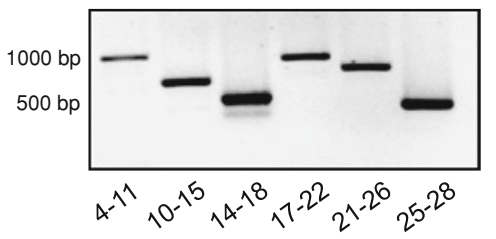

Fig. 1 mRNA expression profiles of nNOS isoforms in the human vastus lateralis (VL) muscle. a A representative agarose gel after RTPCR on biopsies of human VL using nNOS-specific forward primers for the human exons $1 \mathrm{a}, 1 \mathrm{c}, 1 \mathrm{f}$ and $1 \mathrm{~g}$ and a reverse primer for exon 5 . A cDNA band (1,500 base-pairs in length) was amplified only when using exon $1 \mathrm{c}$ as the forward primer. b Evaluation of the mRNA expression patterns of $\mathrm{nNOS}$ isoforms using overlapping primers from exons 4 to 28 . The identity of each band was confirmed by nucleotide sequencing. Note that two bands were amplified when using the nucleotide sequence between exons 14 and 18

the six primer-pair combinations, a single cDNA band was amplified by RT-PCR (Fig. 1b). This finding indicates that no alternative splicing of the mRNA for nNOS occurs between exons 4-15 and exons 17-28. The molecularweight characteristics of the cDNA bands indicated that they contained no introns; nucleotide sequencing confirmed this to be the case (data not shown). When using a primer pair that covered the nNOS nucleotide sequence between exons 14 and 18, two cDNA bands were amplified by RT-PCR (Fig. 1b); each encoded an nNOS isoform with a 14-15-16-17-18 exon-composition, as determined by nucleotide sequencing (data not shown). In the larger molecular-weight band, an additional nucleotide segment, 102 base-pairs in length (exon mu), was inserted between exons 16 and 17. Thus, two mRNA isoforms of nNOS are co-expressed in the human VL. Both variants contain exons $1 \mathrm{c}$ and 2 and are thus alpha isoforms; they differ in the absence or the presence of the exon mu. Since the larger molecular-weight band had an approximately tenfold higher optical density than the smaller one, the mu isoform of nNOS is more abundantly expressed in human VL than the non-mu variant.

\section{Angiogenesis as a function of $\mathrm{nNOS}$ expression} and exercise in human VL biopsies

The quantification analysis of VL biopsies in the light microscope revealed exercise to elicit significant $(P \leq 0.05)$ and concomitant increases in the $\mathrm{C} / \mathrm{F}$-ratio $(21-31 \%)$ and the numerical density of capillaries (4-42\%) in five of the ten individuals, who were assigned to the so-called "angiogenesis responder" (AR) group (Table 1). In the other five subjects [assigned to the "non-angiogenesis responder" (NR) group comprising the subjects that showed no numerical increase in capillarization with training], the pre- and post-exercise values for these parameters did not differ significantly from each other $(P>0.05)$.

Table 1 Morphometric data relating to structural parameters in the human VL biopsies before and after moderate long-term exercise

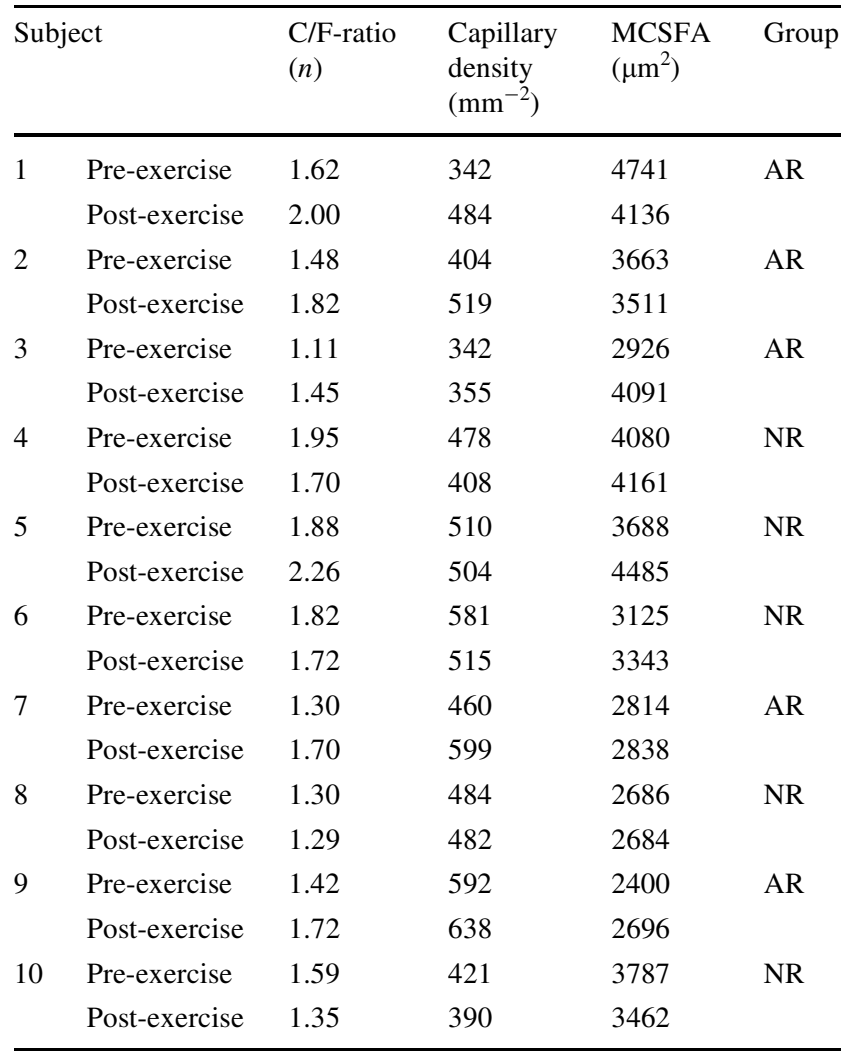

Subjects in whom both the C/F-ratio and the capillary density were concomitantly increased after the exercise were assigned to the angiogenesis responder (AR) group, and those in whom no concomitant exercise-induced increases in these parameters occurred to the group showing no numerical increase in capillarization with training (NR)

$C / F$-ratio number of capillaries per number of fibres, capillary density number of capillaries per skeletal muscle area, MCSFA mean crosssectional fibre area

The mitochondrial volume density which is usually increased after endurance exercise as an established hallmark of the oxidative metabolism was higher after training $(+14 \% ; P<0.05$; AR: $+14 \%$ and NR: $+13 \%)$ and non-significantly related to $\mathrm{VO}_{2} \max (r=0.2 ; P>0.05)$ and capillarity $(r=0.2 ; P>0.05)$ and nNOS mRNA levels $(r=0.4$; $P>0.05)$, if all 10 subjects of the study were included in the analysis (data not shown). The corresponding correlation coefficients for the AR and NR groups are: oxidative capacity and $\mathrm{VO}_{2}$ max: AR: $r=0.50 ; P>0.05$ and NR: $r=0.42 ; P>0.05$; oxidative capacity and capillarity: AR: $r=0.30 ; P>0.05$ and NR: $r=0.53 ; P>0.05$; oxidative capacity and nNOS mRNA expression: AR: $r=0.67$; $P>0.05$ and NR: $r=0.72 ; P>0.05$. Albeit the mitochondrial volume density was increased after endurance exercise, it was not significantly related to nNOS expression in both the AR and the NR cohorts. 


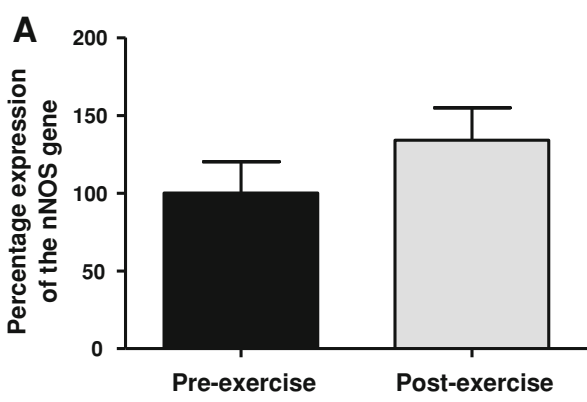

Fig. 2 Exercise-induced up-regulation of the nNOS mRNA expression in VL biopsies derived from subjects of the angiogenesis responder (AR) group but not from the group showing no numerical increase in capillarization with training (NR). a When the group of subjects was considered as a whole, the mRNA levels of nNOS (determined by the real-time PCR technique) were not significantly elevated after exercise

The real-time PCR analysis of the VL biopsies disclosed training to elicit no significant increase $(+34 \% ; P>0.05)$ in the mRNA level of nNOS for the group of participants as a whole (Fig. 2a). However, when the AR group was analysed separately, the nNOS gene revealed to be significantly up-regulated $(+128 \% ; P \leq 0.05)$ in response to exercise. In the NR group, the mRNA level of nNOS was not significantly increased $(-2 \% ; P>0.05)$ by exercise (Fig. 2b). Quantifying the mRNA levels in the biopsies collected before and after the training period, VEGF-A expression was only non-significantly altered if referred to all 10 subjects, those of the AR or the NR group (data not shown). As shown in Fig. 3, a significant correlation existed between the exercise-induced elevation in the mRNA level of nNOS and the exercise-induced increase in the C/F-ratio $(r=0.8 ; P \leq 0.01)$. The nNOS expression was also positively but non-significantly correlated to $\mathrm{VO}_{2} \max (r=0.2$; $P>0.05)$ and mitochondrial volume density $(r=0.4$; $P>0.05$; data not shown).

Conducting immunoblotting, nNOS is predominantly demonstrated as a $165 \mathrm{kDa}$ band (Fig. 4a). If the VL biopsies were subjected to quantitative immunoblotting, the

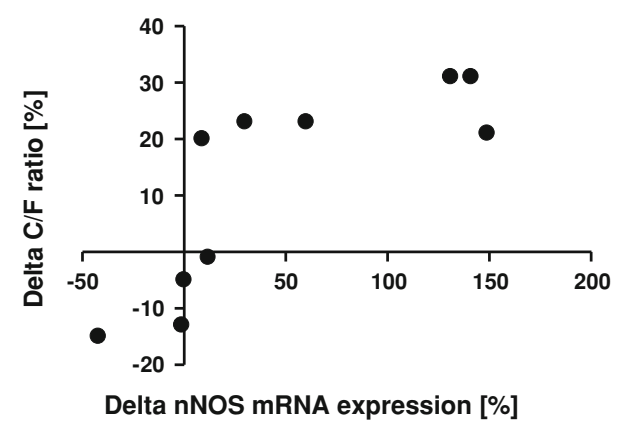

Fig. 3 Relationship between the exercise-induced differences in C/Fratio and mRNA levels of nNOS in human VL biopsies. A significant positive correlation $(r=0.8 ; P \leq 0.01)$ existed between the exerciseinduced differences in C/F-ratio and nNOS expression

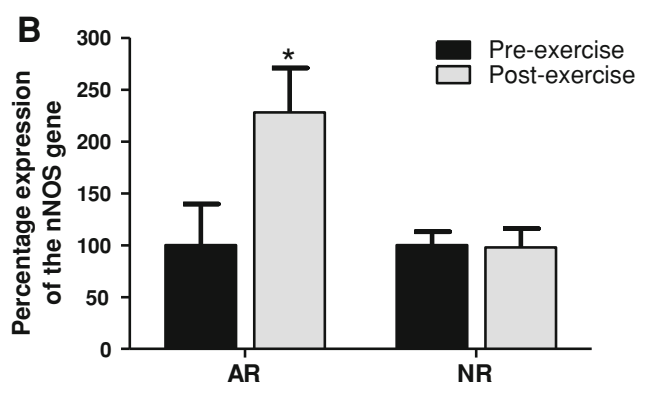

$(+34 \%, P>0.05)$. b In the AR group subjects, exercise induced a significant elevation in the mRNA level of nNOS $(+128 \%, P \leq 0.05)$, whereas in the NR group of individuals, this parameter was not significantly influenced by the training regime $(-2 \%, P>0.05)$. Mean values \pm SEM. a $n=10 ; \mathbf{b} n=5$ in each of the two groups. The preexercise values were set as $100 \%$

nNOS protein levels were significantly higher in the samples collected after than before the exercise in the subjects of the AR group $(+82 \% ; P \leq 0.05)$ but not in those of the NR group $(-16 \% ; P>0.05)$, as shown in Fig. $4 \mathrm{~b}$. The levels of nNOS protein were positively correlated to $\mathrm{C} / \mathrm{F}-$ ratio $(r=0.52 ; P \leq 0.05 ;$ AR: $r=0.21 ; \mathrm{NR}: r=0.38)$ and to those of nNOS mRNA $(r=0.86 ; P \leq 0.01$; AR: $r=0.66$; NR: $r=0.66$ ).

\section{Discussion}

Since the signalling enzyme nNOS undergoes alternative splicing in many tissues (Wang et al. 1999), we first wished to identify its isoform-specific expression pattern in the human VL. The RT-PCR analysis revealed the expression of two mRNA isoforms in these samples. Both mRNA variants express exon 2 and are thus nNOS alpha isoforms; they differ in the absence or presence of exon mu, which inserts between exons 16 and 17 and consists of 102 base-pairs (Silvagno et al. 1996). This finding indicates that exon 2 and exon mu might be simultaneously co-expressed within an individual mRNA molecule of nNOS. Consequently, alpha and mu isoforms of nNOS do not represent different proteins, as has been previously contended (Bradley et al. 2007; Laine and de Montellano 1998). In the real-time PCR analysis, we did not discriminate between the various isoforms of nNOS since the variants do not differ in either their catalytic properties or their sites of localization (Silvagno et al. 1996).

In five of the ten individuals, the $\mathrm{C} / \mathrm{F}$-ratio and the numerical density of capillaries were concomitantly higher after than before exercise, thereby indicating that the absolute number of capillaries increased without a corresponding increase in the mean fibre cross-sectional area (Hudlicka 1998). Hence, in these individuals (assigned to the AR group), the $\mathrm{VL}$ responded to the moderate regime of 


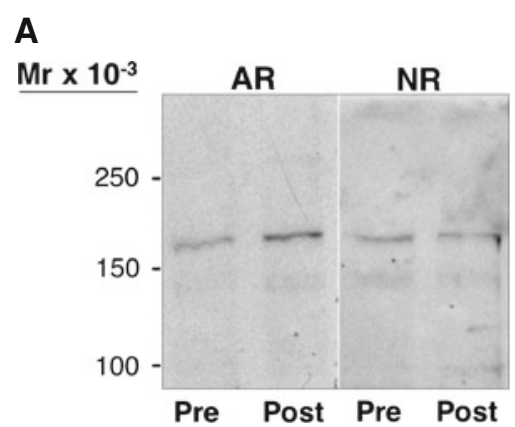

Fig. 4 Exercise-induced up-regulation of the nNOS expression at the protein level in VL biopsies derived from angiogenesis responder (AR) group but not from the group showing no numerical increase in capillarization with training (NR). a Quantitative immunoblotting revealed the nNOS band ( $165 \mathrm{kDa}$ ) to be markedly up-regulated in the VL muscle biopsies of individuals of the AR but not the NR group collected
B

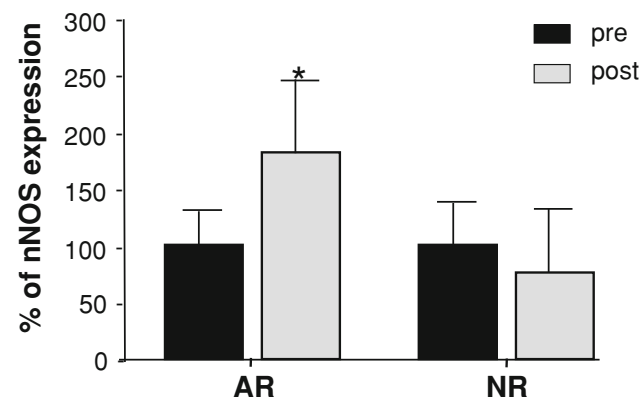

after (post) than before (pre) the exercise. b In the AR group subjects, exercise induced a significant elevation in the protein level of nNOS $(+82 \%, P \leq 0.05)$, whereas in the NR group of individuals, this parameter was not significantly influenced by the training regime $(-16 \%, P>0.05)$. Mean values \pm SEM. $n=4$ in each of the two groups. The pre-exercise values were set as $100 \%$ exercise with a process of angiogenesis. In the other five subjects (assigned to the NR group), a similar regime of exercise elicited no significant change in either the C/Fratio or the numerical density of capillaries. According to the available documentation, the 10 subjects showed different responses in capillarity, although they have underwent a training of identical intensity and duration. The best way to converge these observations is to hypothesize that the individuals exhibited a different degree of malleability. Such a heterogeneous response to the same training stimulus is a well-documented phenomenon (Bouchard and Rankinen 2001; Bray et al. 2009; Timmons and Sundberg 2006), and is presumed to reflect individual differences in genetic predisposition. The overtly non-consistent reactivity of the capillary system might also be influenced by the assortment of the study participants (rather untrained) and the training load (rather moderate), because an induction of angiogenesis in skeletal muscle is observed usually in response to endurance exercise (Gavin 2009).

The real-time PCR analysis revealed a significant exercise-induced increase in the mRNA level of nNOS only in the AR group of individuals, and in these subjects, this parameter was significantly positively correlated with the capillarity. Accordingly, the nNOS protein expression was significantly increased in the VL muscle biopsies of specifically those subjects that underwent angiogenesis as determined by quantitative immunoblotting. On the basis of these findings, we conclude that the expression of nNOS increases at the mRNA and protein level only if the VL responds to exercise with angiogenesis. Correspondingly, increases of $\mathrm{C} /$ F-ratio were accompanied by an up-regulation of sarcolemmal nNOS expression in response to exercise performed during bed rest (Rudnick et al. 2004; Salanova et al. 2008).

In two previously published studies, exercise-induced changes in the protein concentration of nNOS within homoge- nates of VL biopsies that had been derived from subjects with anthropometrically similar characteristics to ours were investigated by immunoblotting (Frandsen et al. 2000; McConell et al. 2007). In one of these studies (Frandsen et al. 2000), the protein levels of nNOS and its catalytic activity were non-significantly up-regulated in response to exercise, whereas in the other (McConell et al. 2007), a 10-day, high-intensity training regime elicited a significant $29 \%$ increase in the protein levels of nNOS. The evidently contradictory observations made in these two studies can be merged with the conclusion that the expression of nNOS increases in human VL muscle in response to endurance exercise only if angiogenesis is simultaneously noticeable, as we have drawn in this investigation.

However, our experimental data do not permit us to ascertain whether the regulation of nNOS expression is a cause or consequence of angiogenesis, or indeed an independent coincidence. Although animal studies demonstrated an up-stream signalling function of higher available levels of nNOS-derived NO on exercise-induced angiogenesis in skeletal muscle (Vassilakopoulos et al. 2003; Williams et al. 2006), it remains to be clarified whether nNOS exerts such a function in humans.

Two explanations of the influence of nNOS on exerciseinduced angiogenesis in skeletal muscle are plausible. According to the first, higher concentrations of nNOS-generated NO would directly or indirectly raise the expression or activity of PGC-1alpha inside the skeletal muscle fibres, which, as an autocrine signalling molecule, would regulate not only mitochondrial biogenesis but also VEGF-Adependent angiogenesis in response to exercise (Arany et al. 2008; Chinsomboon et al. 2009; Leick et al. 2009). Because VEGF-A mRNA is only transiently induced in adaptation to exercise (Gustafsson et al. 2007), we suggest that its expression was already down-regulated at the time point the biopsies were taken. 
Alternatively, since all alpha isoforms of nNOS (either with or without exon mu) are localized to the sarcolemma, the NO-product of their catalysis diffuses into the extramysial milieu, where, as a paracrine signalling molecule, it would influence the contractility of vascular smooth muscle cells (Lau et al. 2000). In consequence to this supposition, up-regulated levels of the alpha isoforms of nNOS would also increase the endomysial availability of $\mathrm{NO}$, thereby enhancing the vasodilation of larger feeding vessels (Sarelius and Pohl 2010). The consequent increase in blood flow would then raise the shear stress in the downstream capillaries. This mechanical signal would represent a relevant one for angiogenesis (Baum et al. 2004), which is triggered by endothelial VEGF-A (Da Silva-Azevedo et al. 2002). According to this assumed sequence of events, nNOS would indirectly influence the degree of traininginduced skeletal muscle angiogenesis by promoting vasodilation. In contrast, the chemical inhibition of nNOS activity did not influence the muscle hyperaemic response to exercise in rats (Copp et al. 2010) underling that the relationship of nNOS/vasodilation in skeletal muscle has to be further investigated.

Consistent with this hypothesis, the loss of sarcolemmal nNOS from the dystrophic muscles of Duchenne muscular dystrophy patients, as well as from the skeletal muscles of mdx- and nNOS-knockout mice, was accompanied by exaggerated fatigue after exercise (Kobayashi et al. 2008; Percival et al. 2008; Wehling-Henricks et al. 2009). In the light of the data gleaned from these loss-of-function studies in dystrophic muscles, our observation that nNOS is up-regulated after training might represent an additional evidence for the functional impact of up-regulated nNOS on exercise-induced angiogenesis in the skeletal muscles of healthy subjects.

Acknowledgments This study was funded by grants from the Swiss National Science Foundation (SNF, 320030-120269) and the Swiss Foundation for Research on Muscle Diseases (SSEM). We would like to thank Franziska Graber and Adolfo Adriozola for their skilful technical support, and Matthias Müller and Fabio Breil for their helpful discussions.

Conflict of interest There is no conflict of interest.

\section{References}

Arany Z, Foo SY, Ma Y, Ruas JL, Bommi-Reddy A, Girnun G, Cooper M, Laznik D, Chinsomboon J, Rangwala SM, Baek KH, Rosenzweig A, Spiegelman BM (2008) HIF-independent regulation of VEGF and angiogenesis by the transcriptional coactivator PGC1alpha. Nature 451:1008-1012

Baum O, Da Silva-Azevedo L, Willerding G, Wöckel A, Planitzer G, Gossrau R, Pries AR, Zakrzewicz A (2004) Endothelial NOS is main mediator for shear stress-dependent angiogenesis in skeletal muscle after prazosin administration. Am J Physiol Heart Circ Physiol 287:H2300-H2308
Bouchard C, Rankinen T (2001) Individual differences in response to regular physical activity. Med Sci Sports Exerc 33:446-451

Bradley SJ, Kingwell BA, Canny BJ, McConell GK (2007) Skeletal muscle neuronal nitric oxide synthase micro protein is reduced in people with impaired glucose homeostasis and is not normalized by exercise training. Metabolism 56:1405-1411

Bray MS, Hagberg JM, Perusse L, Rankinen T, Roth SM, Wolfarth B, Bouchard C (2009) The human gene map for performance and health-related fitness phenotypes: the 2006-2007 update. Med Sci Sports Exerc 41:35-73

Chinsomboon J, Ruas J, Gupta RK, Thom R, Shoag J, Rowe GC, Sawada N, Raghuram S, Arany Z (2009) The transcriptional coactivator PGC-1alpha mediates exercise-induced angiogenesis in skeletal muscle. Proc Natl Acad Sci U S A 106:21401-21406

Coffey VG, Hawley JA (2007) The molecular bases of training adaptation. Sports Med 37:737-763

Copp SW, Hirai DM, Schwagerl PJ, Musch TI, Poole DC (2010) Effects of neuronal nitric oxide synthase inhibition on resting and exercising hindlimb muscle blood flow in the rat. J Physiol 588:1321-1331

Da Silva-Azevedo L, Baum O, Zakrzewicz A, Pries AR (2002) Vascular endothelial growth factor is expressed in endothelial cells isolated from skeletal muscles of nitric oxide synthase knockout mice during prazosin-induced angiogenesis. Biochem Biophys Res Commun 297:1270-1276

Da Silva-Azevedo L, Jahne S, Hoffmann C, Stalder D, Heller M, Pries AR, Zakrzewicz A, Baum O (2009) Up-regulation of the peroxiredoxin- 6 related metabolism of reactive oxygen species in skeletal muscle of mice lacking neuronal nitric oxide synthase. J Physiol 587:655-668

Egginton S (2009) Invited review: activity-induced angiogenesis. Pflugers Arch 457:963-977

Frandsen U, Hoffner L, Betak A, Saltin B, Bangsbo J, Hellsten Y (2000) Endurance training does not alter the level of neuronal nitric oxide synthase in human skeletal muscle. J Appl Physiol 89:1033-1038

Gavin TP (2009) Basal and exercise-induced regulation of skeletal muscle capillarization. Exerc Sport Sci Rev 37:86-92

Gustafsson T, Rundqvist H, Norrbom J, Rullman E, Jansson E, Sundberg CJ (2007) The influence of physical training on the angiopoietin and VEGF-A systems in human skeletal muscle. J Appl Physiol 103:1012-1020

Hoppeler H, Howald H, Conley K, Lindstedt SL, Claassen H, Vock P, Weibel ER (1985) Endurance training in humans: aerobic capacity and structure of skeletal muscle. J Appl Physiol 59:320-327

Hoppeler H, Klossner S, Fluck M (2007) Gene expression in working skeletal muscle. Adv Exp Med Biol 618:245-254

Hudlicka O (1998) Is physiological angiogenesis in skeletal muscle regulated by changes in microcirculation? Microcirculation 5:7-23

Hudlicka O, Brown MD (2009) Adaptation of skeletal muscle microvasculature to increased or decreased blood flow: role of shear stress, nitric oxide and vascular endothelial growth factor. J Vasc Res 46:504-512

Hudlicka O, Brown MD, Silgram H (2000) Inhibition of capillary growth in chronically stimulated rat muscles by N(G)-nitro-Larginine, nitric oxide synthase inhibitor. Microvasc Res 59:45-51

Jackson MJ, Pye D, Palomero J (2007) The production of reactive oxygen and nitrogen species by skeletal muscle. J Appl Physiol 102:1664-1670

Kobayashi YM, Rader EP, Crawford RW, Iyengar NK, Thedens DR, Faulkner JA, Parikh SV, Weiss RM, Chamberlain JS, Moore SA, Campbell KP (2008) Sarcolemma-localized nNOS is required to maintain activity after mild exercise. Nature 456:511-515

Kobzik L, Reid MB, Bredt DS, Stamler JS (1994) Nitric oxide in skeletal muscle. Nature 372:546-548 
Laine R, de Montellano PR (1998) Neuronal nitric oxide synthase isoforms alpha and mu are closely related calpain-sensitive proteins. Mol Pharmacol 54:305-312

Lau KS, Grange RW, Isotani E, Sarelius IH, Kamm KE, Huang PL, Stull JT (2000) nNOS and eNOS modulate cGMP formation and vascular response in contracting fast-twitch skeletal muscle. Physiol Genomics 2:21-27

Leick L, Hellsten Y, Fentz J, Lyngby SS, Wojtaszewski JF, Hidalgo J, Pilegaard H (2009) PGC-1alpha mediates exercise-induced skeletal muscle VEGF expression in mice. Am J Physiol Endocrinol Metab 297:E92-E103

McConell GK, Bradley SJ, Stephens TJ, Canny BJ, Kingwell BA, Lee-Young RS (2007) Skeletal muscle nNOS mu protein content is increased by exercise training in humans. Am J Physiol Regul Integr Comp Physiol 293:R821-R828

Melikian N, Seddon MD, Casadei B, Chowienczyk PJ, Shah AM (2009) Neuronal nitric oxide synthase and human vascular regulation. Trends Cardiovasc Med 19:256-262

Percival JM, Anderson KN, Gregorevic P, Chamberlain JS, Froehner SC (2008) Functional deficits in nNOSmu-deficient skeletal muscle: myopathy in nNOS knockout mice. PLoS One 3:e3387

Rudnick J, Puttmann B, Tesch PA, Alkner B, Schoser BG, Salanova M, Kirsch K, Gunga HC, Schiffl G, Luck G, Blottner D (2004) Differential expression of nitric oxide synthases (NOS 1-3) in human skeletal muscle following exercise countermeasure during 12 weeks of bed rest. FASEB J 18:1228-1230

Salanova M, Schiffl G, Puttmann B, Schoser BG, Blottner D (2008) Molecular biomarkers monitoring human skeletal muscle fibres and microvasculature following long-term bed rest with and without countermeasures. J Anat 212:306-318
Sarelius I, Pohl U (2010) Control of muscle blood flow during exercise: local factors and integrative mechanisms. Acta Physiol (Oxf) 199:349-365

Silvagno F, Xia H, Bredt DS (1996) Neuronal nitric-oxide synthase$\mathrm{mu}$, an alternatively spliced isoform expressed in differentiated skeletal muscle. J Biol Chem 271:11204-11208

Stamler JS, Meissner G (2001) Physiology of nitric oxide in skeletal muscle. Physiol Rev 81:209-237

Suter E, Hoppeler H, Claassen H, Billeter R, Aebi U, Horber F, Jaeger P, Marti B (1995) Ultrastructural modification of human skeletal muscle tissue with 6-month moderate-intensity exercise training. Int J Sports Med 16:160-166

Timmons JA, Sundberg CJ (2006) Oligonucleotide microarray expression profiling: human skeletal muscle phenotype and aerobic exercise training. IUBMB Life 58:15-24

Vassilakopoulos T, Deckman G, Kebbewar M, Rallis G, Harfouche R, Hussain SN (2003) Regulation of nitric oxide production in limb and ventilatory muscles during chronic exercise training. Am J Physiol Lung Cell Mol Physiol 284:L452-L457

Wang Y, Newton DC, Robb GB, Kau CL, Miller TL, Cheung AH, Hall AV, VanDamme S, Wilcox JN, Marsden PA (1999) RNA diversity has profound effects on the translation of neuronal nitric oxide synthase. Proc Natl Acad Sci U S A 96:12150-12155

Wehling-Henricks M, Oltmann M, Rinaldi C, Myung KH, Tidball JG (2009) Loss of positive allosteric interactions between neuronal nitric oxide synthase and phosphofructokinase contributes to defects in glycolysis and increased fatigability in muscular dystrophy. Hum Mol Genet 18:3439-3451

Williams JL, Cartland D, Hussain A, Egginton S (2006) A differential role for nitric oxide in two forms of physiological angiogenesis in mouse. J Physiol 570:445-454 